Article

\title{
Engineered Glucose Oxidase Capable of Quasi-Direct Electron Transfer after a Quick-and-Easy Modification with a Mediator
}

\author{
Nanami Suzuki ${ }^{1,+}{ }^{\text {, Jinhee Lee }}{ }^{2,+} \oplus$, Noya Loew ${ }^{2}$, Yuka Takahashi-Inose ${ }^{3}$, \\ Junko Okuda-Shimazaki ${ }^{2}$, Katsuhiro Kojima ${ }^{3}$, Kazushige Mori ${ }^{3}$, Wakako Tsugawa ${ }^{1}$ \\ and Koji Sode ${ }^{2, *}$ \\ 1 Department of Biotechnology and Life Science, Graduate School of Engineering, Tokyo University of \\ Agriculture and Technology, 2-24-16 Naka-cho, Koganei, Tokyo 184-8588, Japan; \\ nsuzuki@protonmail.com (N.S.); tsugawa@cc.tuat.ac.jp (W.T.) \\ 2 Joint Department of Biomedical Engineering, The University of North Carolina at Chapel Hill and North \\ Carolina State University, Chapel Hill, NC 27599, USA; jh.lee@unc.edu (J.L.); noya-loew@rs.tus.ac.jp (N.L.); \\ jokudas@email.unc.edu (J.O.-S.) \\ 3 Ultizyme International Ltd., 3-9-5. Taihei, Sumida, Tokyo 130-0012, Japan; yukappe1122@gmail.com (Y.T.-I.); \\ katsuhiro.kojima@gmail.com (K.K.); mori_ka1213@yahoo.co.jp (K.M.) \\ * Correspondence: ksode@email.unc.edu; Tel.: +1-919-966-3550 \\ + These authors contributed equally to this work.
}

Received: 3 January 2020; Accepted: 5 February 2020; Published: 8 February 2020

check for updates

\begin{abstract}
Glucose oxidase (GOx) has been widely utilized for monitoring glycemic levels due to its availability, high activity, and specificity toward glucose. Among the three generations of electrochemical glucose sensor principles, direct electron transfer (DET)-based third-generation sensors are considered the ideal principle since the measurements can be carried out in the absence of a free redox mediator in the solution without the impact of oxygen and at a low enough potential for amperometric measurement to avoid the effect of electrochemically active interferences. However, natural GOx is not capable of DET. Therefore, a simple and rapid strategy to create DET-capable GOx is desired. In this study, we designed engineered GOx, which was made readily available for single-step modification with a redox mediator (phenazine ethosulfate, PES) on its surface via a lysine residue rationally introduced into the enzyme. Thus, PES-modified engineered GOx showed a quasi-DET response upon the addition of glucose. This strategy and the obtained results will contribute to the further development of quasi-DET GOx-based glucose monitoring dedicated to precise and accurate glycemic control for diabetic patient care.
\end{abstract}

Keywords: glucose oxidase; direct electron transfer; amine-reactive phenazine ethosulfate; glucose sensor; glycemic level monitoring

\section{Introduction}

Glucose oxidase ( $\beta$-D-glucose: oxygen 1-oxidoreductases, E.C. 1.1.3.4, GOx) is a flavoprotein that catalyzes the oxidation of $\beta$-D-glucose at its hydroxyl group linked to the carbon one using molecular oxygen as the electron acceptor to produce D-glucono-1,5-lactone and hydrogen peroxide (Scheme 1). Since the first report by Clark and Lyons on an enzyme electrode employing GOx and an oxygen electrode for glucose monitoring [1], extensive studies have been carried out to develop improved enzyme-based systems for monitoring glycemic levels. The reason for the popularity of GOx lies in its commercial availability, high activity, and substrate specificity against glucose. 


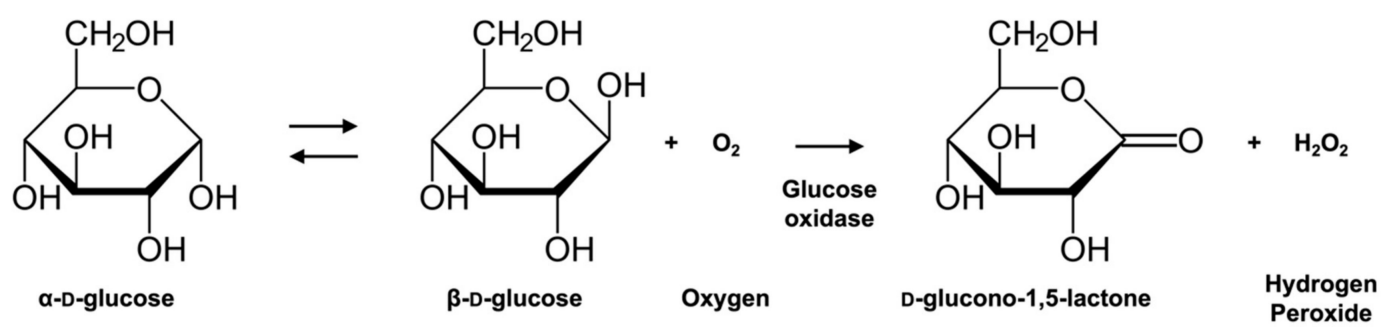

Scheme 1. Reaction scheme of glucose oxidation catalyzed by glucose oxidase.

Electrochemical glucose biosensors can be divided into three principles (Scheme 2). The "first-generation" glucose sensing principle utilizes molecular oxygen as the primary electron acceptor, and the glucose concentration is determined by the consumption of oxygen or generation of hydrogen peroxide. In the "second-generation" principle, artificial electron acceptors (also referred to as electron mediators) replace oxygen in the reaction [2], which enables avoiding the production of harmful hydrogen peroxide. Furthermore, due to the lower redox potential compared to that of hydrogen peroxide, the use of certain mediators enables us to decrease the applied potential for biosensors based on glucose oxidase. However, when oxidase is employed, this reaction is inherently affected by the oxygen concentration in a sample. Accordingly, glucose dehydrogenase (GDH) has become the major enzyme for glucose sensing in the second-generation principle. However, because of GDH's broad substrate specificity, which can lead to potentially fatal errors in glucose sensing [3], GOx has still been the focus due to its high specificity. Due to the advantages of GOx over GDH, studies are ongoing to engineer GOx into dehydrogenase [4-7]. In the "third-generation" sensor principle, glucose dehydrogenases capable of direct electron transfer (DET) are employed. Thereby, electrons derived from glucose oxidation are transferred directly to the electrode. Glucose sensor employing DET-type enzymes has several advantages comparing mediator type glucose sensors, e.g., a smaller number of essential elements for sensing, a smaller number of essential reactions for detection, operation at a lower applying potential than those utilizing hydrogen peroxide, and a major kind of mediator used in second-generation principle such as ferrocene, ferricyanide, and ruthenium complexes [8,9]. This low-applying potential contributes to eliminate interference by electrochemically active ingredients in the samples. Therefore, the DET-based enzyme sensor principle has been considered ideal for redox enzyme-based electrochemical sensors.

Considering the superior enzymatic property of the "gold standard," several efforts have been made to realize DET GOx-based glucose sensing. However, the 3D structure of GOx revealed that the redox cofactor, flavin adenine dinucleotide (FAD) is buried deeply within the protein. In addition, previous reports $[10,11]$ attributed the voltammograms associated with GOx to free FAD, which suggests that only the free FAD of GOx showed voltammetric signals, whereas bound FAD did not, and bound FAD undergoes electron transfer at a more positive potential not observed in voltammetry [12]. Therefore, the only approach that realizes DET for GOx-based glucose sensing is the modification of the redox species onto the enzyme surface. Since the 1980s, GOx modifications by covalently attaching redox mediators have been reported to achieve quasi-DET using derivatives of ferrocene [13-21], phenothiazine [22], ruthenium [23], tetrathiafulvalene [24], and phenoxazine [25] to modify GOx. However, the modification procedure requires complex and laborious steps to introduce functional groups for mediator modification. In addition, no report has demonstrated the rational design of residues where redox mediators should be modified on GOx. 


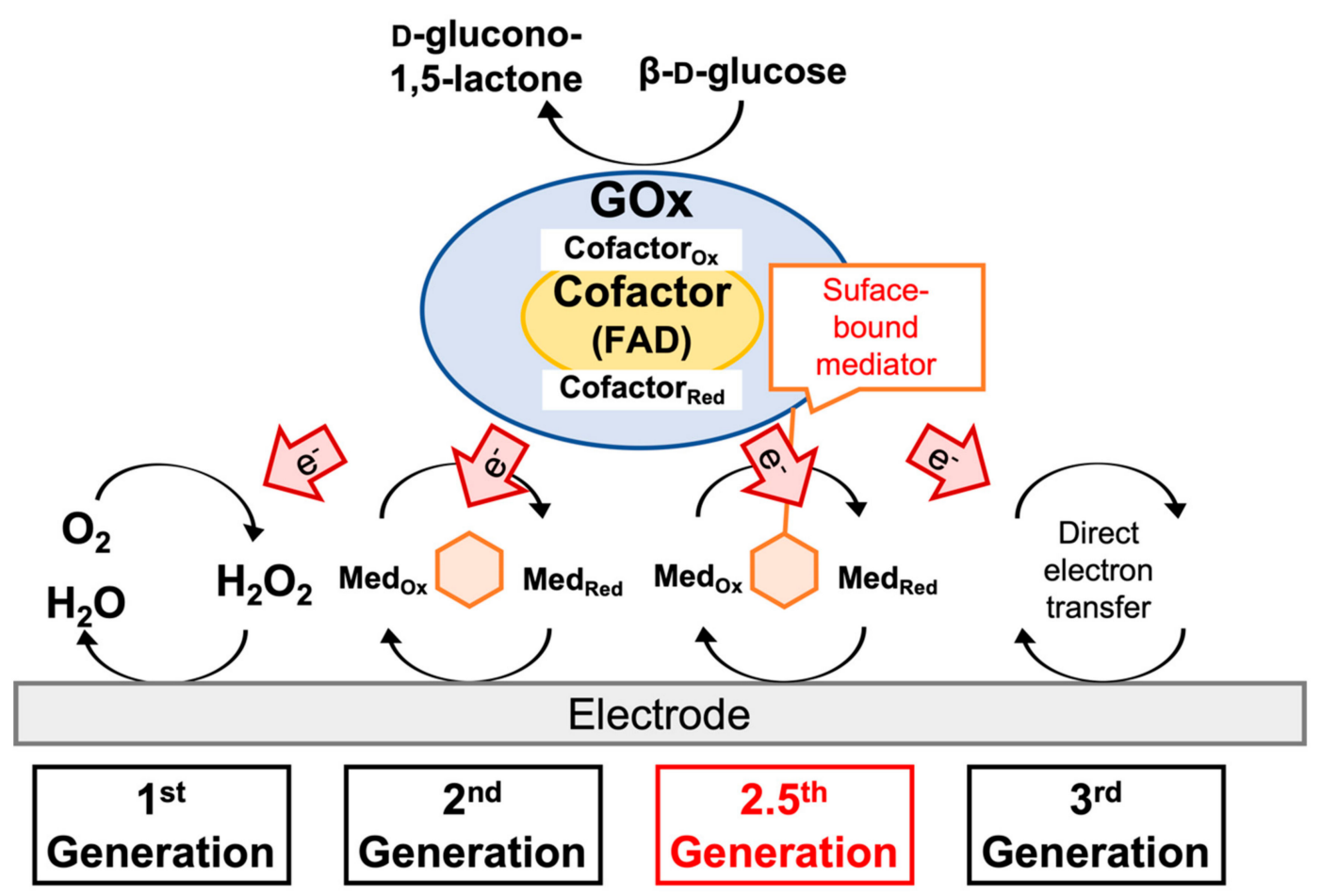

Scheme 2. Schematic illustration of the glucose sensor generations using glucose oxidase (GOx).

To achieve a versatile and simple method for enzyme modification, we previously reported a rapid and single-step conjugation using 1-[3-(succinimidyloxycarbonyl)propoxy]-5-ethylphenazinium trifluoromethanesulfonate (or amine-reactive phenazine ethosulfate; arPES) [26,27] onto the surface of the redox enzyme. The attachment of these redox mediators to redox enzymes enables intramolecular electron transfer from the enzyme redox center to the mediator and intermolecular electron transfer from the mediator to an electrode. This electron transfer via the mediator bound to the enzyme surface can be described as quasi-direct electron transfer (quasi-DET) and "2.5th generation" principle. arPES harbors two parts, i.e., a succinimide group and mediator, namely, methoxy PES, which is a stable, relatively low redox potential mediator. Upon mixing arPES and an enzyme, the primary amine groups on the enzyme attack the carboxylate ester carbon of the succinimide group of arPES and form amide bonds at room temperature.

Therefore, in this study, we aimed to design an engineered GOx suitable for quasi-DET-type sensor applications through the quick and easy modification of mediators using arPES. As the enzyme, GOx from Aspergillus niger was chosen, and amino acid substitution was designed based on the 3D structure to introduce a lysine residue in GOx, which was modified by arPES. After investigating the characteristics of the engineered GOx, PES-modified GOx was prepared, and a quasi-DET-type glucose sensor was constructed.

\section{Results and Discussion}

\subsection{Engineering of GOx for PES Modification}

2.1.1. Investigation of the Availability of Wild-Type Aspergillus niger-Derived GOx for Redox Mediator Modification

First, we investigated whether wild-type GOx from A. niger (AnGOx-WT) is readily available for modification by PES to show a quasi-DET response. AnGOx-WT was modified with PES, and the dye-mediated dehydrogenase activity was determined with an electron mediator, phenazine methosulfate (PMS), and a terminal electron acceptor, 3-(4,5-dimethyl-2thiazolyl)-2,5-diphenyl-2H-tetrazolium bromide (MTT). When PMS and MTT were used together 
(PMS/MTT system), the PMS functioned as the primary electron acceptor of the enzyme and mediated electrons to the bulky MTT. We previously reported that MTT alone did not function as the electron acceptor for a variety of redox enzymes. However, the presence of PMS facilitated the reduction of MTT by the oxidation substrate [26]. Considering that both PMS and MTT are negatively charged in their oxidative status, we assumed that the low reactivity of MTT as the primary electron acceptor might be attributed to its bulkiness when compared with PMS. Therefore, we have used the availability of MTT as the sole electron acceptor in the solution (MTT system) as the indication that PES was successively modified on the surface of redox enzymes, where internal electron transfer from a deeply buried cofactor to PES occurred. Consequently, bulky MTT can be reduced [26]. Since GOx was revealed that it did not utilize MTT as the primary electron acceptor, we followed our previous empirical experimental condition to analyze the modification of PES on the surface of GOx and evaluate the availability of the arPES modification procedure to prepare quasi-DET type GOx. We assumed that successive PES modification might enable PES-modified GOx to use MTT as the primary electron acceptor to show dye-mediated glucose dehydrogenase activity.

The results are shown in Table 1. The PES-modified AnGOx-WT revealed almost identical dye-mediated dehydrogenase activity as that of unmodified and PES-modified AnGOx-WT when the PMS/MTT system was employed, which indicates that the PES-modified GOx maintained its original activity. However, in the MTT system, only low dehydrogenase activity was observed after the modification of PES, which was indistinguishable from that of the intact $A n G O x-W T$.

Table 1. Dehydrogenase activity of unmodified and phenazine ethosulfate (PES)-modified glucose oxidases (GOxs) (wild-type; WT and mutant; I489K) determined with the phenazine methosulfate (PMS)/3-(4,5-dimethyl-2-thiazolyl)-2,5-diphenyl-2H-tetrazolium bromide (MTT) system and MTT system.

\begin{tabular}{cccc}
\hline \multirow{2}{*}{ Enzyme } & \multirow{2}{*}{ PES Modification } & \multicolumn{2}{c}{ Dehydrogenase Activity [U/mg] } \\
\cline { 3 - 4 } & & PMS/MTT System & MTT System \\
\hline \multirow{2}{*}{ AnGOx-WT } & - & 16 & n.d.* \\
& + & 18 & $5.3 \times 10^{-2}$ \\
\multirow{2}{*}{ AnGOx-I489K } & - & 25 & $7.5 \times 10^{-3}$ \\
& + & 19 & 10 \\
\hline & & ${ }^{*}$ Not detected.
\end{tabular}

To investigate the presence of the PES molecule on the surface of $A n \mathrm{GOx}-\mathrm{WT}$ after PES modification, we further characterized the enzyme on an electrode. The cyclic voltammograms of PES-modified AnGOx-WT clearly showed oxidation and reduction peaks at approximately $-100 \mathrm{mV}$, which revealed the presence of PES on the surface of the enzyme (Figure S1c), which were not observed from the electrode with PES-unmodified intact AnGOx-WT (Figure S1a). These results indicate that PES was successfully modified on the surface of GOx and was electrochemically active. Considering that no catalytic current was observed in the presence of glucose (Figure S1d), together with the results of the previously mentioned dye-mediated dehydrogenase activity investigations using the MTT system, the position of PES modification on AnGOx-WT was not suitable to accept electrons from its redox cofactor, FAD, in the catalytic center.

\subsubsection{Identification of the Appropriate Site for Substitution with a Lysine Residue}

To locate PES at a suitable position for the electron relay, we designed an additional lysine residue, where arPES should make a covalent bond. To determine the position, we compared the structure of $A n G O x$ (PDB ID: 1CF3) with a model structure of Botryotinia fuckeliana-derived GDH ( $B f G D H)$, which has a structure similar to that of $A n G O x$ but shows a quasi-DET response after PES modification. A closer look at the position of lysine residues in the crystal structures of $A n \mathrm{GOx}$ (1CF3), 
a structural model of $B f G D H$, and the crystal structure of $A$. flavus-derived GDH (AfGDH, PDB ID: $4 \mathrm{YNU})$, on which the model was based, shows that the residues are distributed very differently, despite the similar overall structure (Figure 1). However, there is one notable residue that is located at what appears to be the entrance of a pathway to the catalytic center. This residue is a lysine in both GDHs (K483 in BfGDH, K477 in AfGDH) and an isoleucine in AnGOx (I489). Furthermore, this residue appears to be located close enough to the FAD cofactor to receive electrons. Therefore, this residue appears to be an ideal candidate for attaching PES to function as an electron relay between the FAD and the terminal electron acceptor.

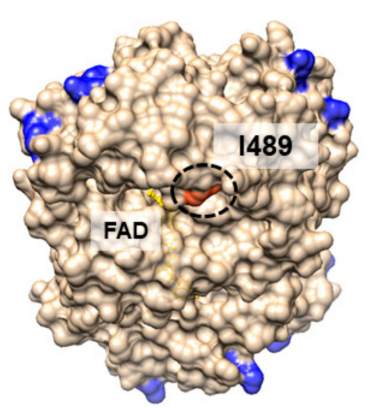

AnGOx (1CF3)

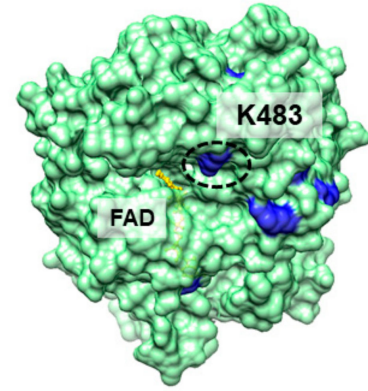

$B f G D H$

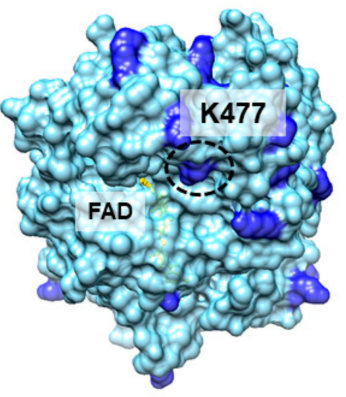

AfGDH

(4YNU)

Figure 1. Comparison of the positions of lysine residues in Aspergillus niger derived glucose oxidase (AnGOx) (PDB ID: 1CF3), Botryotinia fuckeliana derived glucose dehydrogenase (BfGDH) (model), and A.flavus derived GDH (AfGDH) (PDB ID: 4YNU). Lysine residues are shown in dark blue. In BfGDH and $A f G D H$, a lysine residue (K483, K477, circled) is located at the entrance of what appears to be a pathway to the active center. In $A n G O x$, an isoleucine residue (I489, circled) is located at this position.

\subsection{Construction and Characterization of the GOx Mutant for Quasi-DET after PES Modification}

The isoleucine residue I489 in AnGOx was substituted with a lysine residue in the AnGOx-I489K mutant. Kinetic parameters of the mutant are shown in the Supplementary Material (Table S1). Notably, the modification of $A n G O x-I 489 K$ with PES significantly increased the activity value obtained with the MTT system (Table 1), which indicated the successful formation of an electron relay from FAD in the catalytic center to the enzyme surface. Additionally, a similar $K_{\mathrm{m}}$ value was observed with $A n G O x-I 489 \mathrm{~K}$ dehydrogenase activity before and after PES modification (Table S1). Therefore, it appears that the attachment of PES to the substrate entrance did not alter the accessibility of glucose to the active center.

Cyclic voltammograms of PES-modified AnGOx-I489K clearly showed oxidation and reduction peaks at approximately $-100 \mathrm{mV}$, which indicates the presence of PES on the enzyme surface (Figure 2c). In the presence of glucose, a catalytic current, i.e., an increase in the current at potentials higher than that of the oxidation peak of PES, was observed when we used PES-modified AnGOx-I489K (Figure 2d), and no such catalytic current was observed with unmodified AnGOx-I489K (Figure 2b). The observed reduction peak decrease at $-0.4 \mathrm{~V}$ upon addition of glucose, which would be attributed to the decrease of local oxygen concentration caused by oxygen consumption by immobilized GOx. This result confirms that $A n \mathrm{GOx}-\mathrm{I} 489 \mathrm{~K}$ acquired quasi-DET ability after modification with PES. PES bound to the lysine residue at position 489 provided the missing link in the electron relay from FAD to the electrode. 


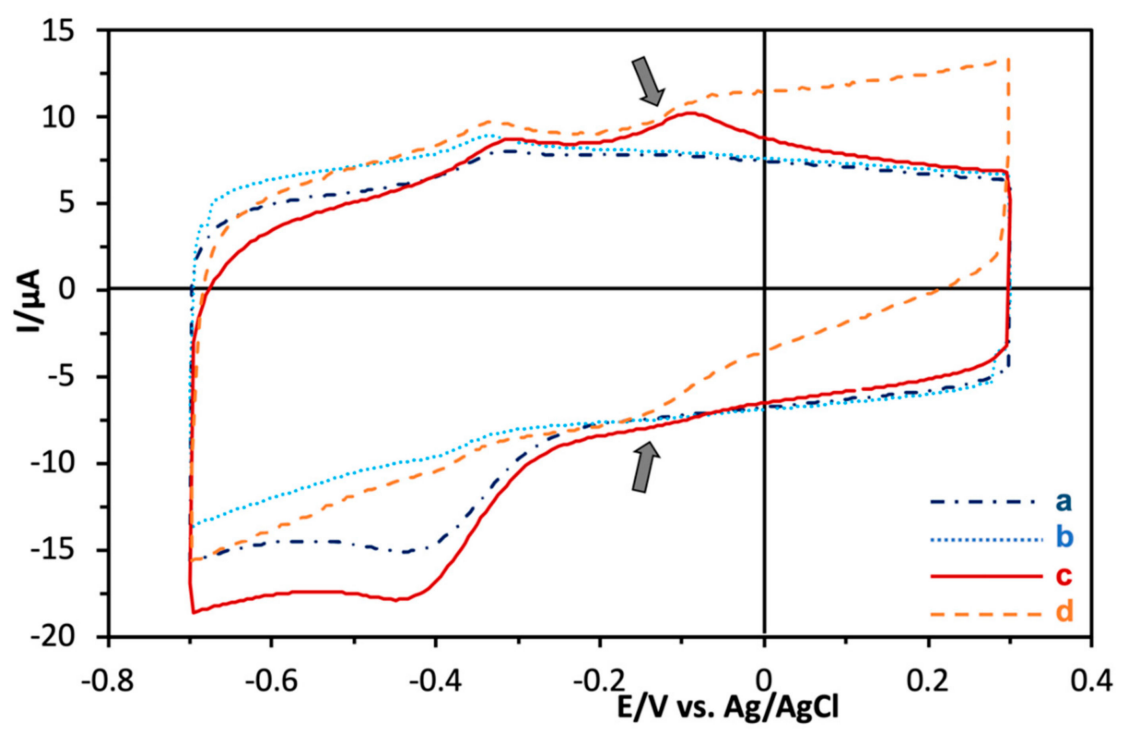

Figure 2. Cyclic voltammograms of (a) unmodified $A n \mathrm{GOx}-\mathrm{I} 489 \mathrm{~K}$ in the absence of glucose. (b) Unmodified $A n G O x-I 489 \mathrm{~K}$ in the presence of $33 \mathrm{mM}$ glucose. (c) PES-modified $A n G O x-I 489 \mathrm{~K}$ in the absence of glucose. (d) PES-modified AnGOx-I489K in the presence of $33 \mathrm{mM}$ glucose. Scan rate $50 \mathrm{mV} / \mathrm{s}$. Arrows: peaks due to PES.

\subsection{Characterization of Electrodes with PES-Modified AnGOx-I489K}

Lastly, we investigated the quasi-DET response against various glucose concentrations. Chronoamperometric measurements using the electrode with immobilized PES-modified AnGOx-I489K showed clear and stable responses for the addition of glucose at an operating potential of $0 \mathrm{mV}$ vs. $\mathrm{Ag} / \mathrm{AgCl}$ (Figure 3A). A plot of the response current against the glucose concentration showed a linear increase until approximately $3 \mathrm{mM}$ and saturation at glucose concentrations higher than approximately $17 \mathrm{mM}$ (Figure 3B). The response on the electrode gave the apparent $K_{\mathrm{m}}$ value of $2.3 \mathrm{mM}$ and $I_{\max }$ value of $1.4 \mu \mathrm{A}$ even though the mutation nor PES modification did not change the $K_{\mathrm{m}}$ value of GOx (Table S1). This smaller apparent $K_{\mathrm{m}}$ value observed in the electrochemical measurement was assumed to be the difference of a rate limiting step. In the homogeneous solution, the reductive half reaction of the cofactor was the rate limiting step. However, in the electrochemical measurement, the electron transfer between quasi-DET GOx and the electrode might be limited. Essentially, the rate limiting step in the electrochemical reaction with PES modified GOx was assumed at the oxidative half reaction where reduced PES was oxidized by the electrode. Therefore, the apparent $K_{\mathrm{m}}$ value observed on the electrode did not match the actual $K_{\mathrm{m}}$ value determined with the redox dye.

The cyclic voltammograms (Figure 2) and chronoamperometric measurements (Figure 3A,B) confirm that PES-modified AnGOx-I489K is capable of quasi-direct electron transfer due to PES modification of the introduced lysine residue. Based on these results, it seems that, after the FAD cofactor is reduced by glucose, it is re-oxidized by the PES bound to the lysine residue at position 489 . The reduced PES is then oxidized directly at the electrode or possibly via other PES molecules bound at the surface of GOx. 
A



B

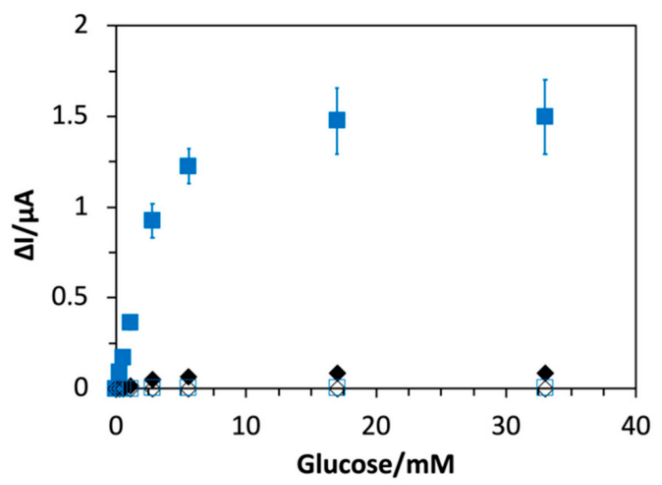

Figure 3. (A) Time courses of chronoamperometric measurements with (a) PES-modified AnGOx-I489K and (b) PES-modified AnGOx-WT. Application potential $0 \mathrm{mV}$ vs. Ag/AgCl. Arrows indicate the addition of glucose. Concentrations over arrows are of the total glucose after addition. (B) Dependence of response current on glucose concentration for PES-modified AnGOx-I489K (filled squares), PES-modified AnGOx-WT (filled diamonds), unmodified $A n G O x-I 489 \mathrm{~K}$ (open squares), and unmodified $A n G O x-W T$ (open diamonds). Application potential $0 \mathrm{mV}$ vs. $\mathrm{Ag} / \mathrm{AgCl}$.

\section{Materials and Methods}

\subsection{Materials}

arPES was kindly donated by Dojindo Laboratories Co. Ltd. (Kumamoto, Japan). Phenazine methosulfate (PMS), $\mathrm{D}(+)$-glucose, potassium dihydrogen phosphate, dipotassium hydrogen phosphate and Triton X-100 were purchased from Kanto Chemical Co. Inc. (Tokyo, Japan). 3-(4,5Dimethyl-2-thiazolyl)-2,5-diphenyl-2H-tetrazolium bromide (MTT) was purchased from Dojindo Laboratories Co. Ltd. D-(+)-trehalose dihydrate and glutaraldehyde were purchased from FUJIFILM Wako Pure Chemical Corporation (Osaka, Japan). $N$-[Tris(hydroxymethyl)methyl]glycine (tricine) was purchased from Sigma-Aldrich Co. LLC (St. Louis, MO, USA). Ketjen black (ECP600JD), was obtained from Mitsubishi Chemical Corporation (Tokyo, Japan).

\subsection{Identification of the Appropriate Mutation Site by Comparing the GOx and GDH Structures}

A 3D structural model of Botryotinia fuckeliana-derived GDH (BfGDH) was generated based on the crystal structure of FAD-dependent glucose dehydrogenase derived from A. flavus (AfGDH, PDB ID: 4YNU) [28]. The amino acid sequences of GOx derived from A. niger (AnGOx, PDB ID: 1CF3) and of the GDHs were aligned using the multiple sequence alignment software ClustalW (http://www.clustal.org) [29]. By comparing the structures of GOx and the GDHs, lysine residues in the GDHs were identified, which are close to what appears to be the entrance of pathways leading to the catalytic center and which have corresponding non-lysine residues in the GOx.

\subsection{Expression Vector Preparation and Recombinant Expression of GOxs}

The structural gene of wild-type GOx was prepared as previously described [7]. The gene was inserted into the multiple cloning site of the expression vector pET30c(+) (Merck KGaA, Darmstadt, Germany). Expression vectors of GOx mutants were prepared by site-directed mutagenesis using the QuikChange Mutagenesis Kit (Agilent Technology Inc., Santa Clara, CA, USA). The correctness of the mutations was confirmed using the ABI Prism 3100 Genetic Analyzer (Applied Biosystems, Foster City, CA, USA). Expression and refolding of GOxs (both wild-type and mutant) were carried out as previously described [30]. 


\subsection{PES Modification of Enzymes}

GOxs (wild-type and mutant) were modified using arPES, as previously described [26] with some changes. GOx and arPES were mixed in $20 \mathrm{mM}$ tricine buffer (pH 8.3) to $13 \mu \mathrm{M}$ (based on catalytic centers) and $2.0 \mathrm{mM}$, respectively, and incubated at $25^{\circ} \mathrm{C}$ for $2 \mathrm{~h}$ while shaking at $1200 \mathrm{rpm}$. Afterward, the sample was ultra-filtered to remove excess arPES and exchange the buffer to $20 \mathrm{mM}$ potassium phosphate buffer (P.P.B.) using Amicon Ultra-0.5 30K centrifugal filters (molecular cut-off, $30 \mathrm{kDa}$ ) (Sigma-Aldrich Co. LLC, St. Louis, MO, USA).

\subsection{Enzyme Activity}

Two types of dehydrogenase activity assays were performed, which were named the PMS/MTT system and MTT system. For the PMS/MTT system, the increase in absorbance at $565 \mathrm{~nm}$ (from the formation of formazan dye due to the reduction of MTT) of a mixture of GOx, $100 \mathrm{mM}$ glucose, $0.6 \mathrm{mM}$ PMS, and $1 \mathrm{mM}$ MTT in $20 \mathrm{mM}$ P.P.B. was monitored. For the MTT system, the increase in absorbance at $565 \mathrm{~nm}$ of a mixture of GOx, $100 \mathrm{mM}$ glucose, and $1 \mathrm{mM}$ MTT in $20 \mathrm{mM}$ P.P.B. was monitored. The reduction of $1 \mu \mathrm{mol} \mathrm{MTT} \mathrm{in} 1 \mathrm{~min}$, corresponding to the oxidation of $1 \mu \mathrm{mol} / \mathrm{min}$ glucose, was defined as $1 \mathrm{U}$ dehydrogenase activity.

\subsection{Preparation of Enzyme Electrodes}

First, $0.54 \mu \mathrm{L}$ of enzyme ink containing $1.5 \mathrm{mg} / \mathrm{mL}$ GOx (unmodified or PES-modified), $0.5 \%$ trehalose, $0.6 \%$ Ketjen black, and 1.2\% Triton X-100 in $20 \mathrm{mM}$ P.P.B. was dropped on a glassy carbon electrode (3.0 mm diameter, BAS Inc., Tokyo, Japan), and dried at $25^{\circ} \mathrm{C}$. Next, the enzyme was cross-linked in glutaraldehyde vapor for $1 \mathrm{~h}$ at room temperature. The electrodes were stored at $25^{\circ} \mathrm{C}$ until use.

\subsection{Electrochemical Evaluation}

Before use, the electrodes were equilibrated in the electrolyte (100 mM P.P.B., pH 7.0) for at least $20 \mathrm{~min}$. A platinum wire (TANAKA Kikinzoku K.K., Tokyo, Japan) was used as a counter electrode and $\mathrm{Ag} / \mathrm{AgCl} / 3 \mathrm{M} \mathrm{NaCl}$ as a reference electrode (BAS Inc., Tokyo, Japan). An SP-150 potentiostat (Bio-Logic Science Instruments, Seyssinet-Pariset, France) was used for electrochemical measurements. Cyclic voltammetry was performed for a potential range of $-0.7 \mathrm{~V}$ to $0.3 \mathrm{~V}$ at a scan rate of $50 \mathrm{mV} / \mathrm{s}$. For the chronoamperometric measurements, a potential of $0 \mathrm{~V}$ was applied. Glucose was added to the final concentrations of $0,0.28,0.56,1.1,2.8,5.6,17$, and $33 \mathrm{mM}$.

\section{Conclusions}

By strategically designing the position of a lysine residue for PES modification by the structural comparison between GOx and FADGDHs, an engineered $A n \mathrm{GOx}-\mathrm{I} 489 \mathrm{~K}$ was constructed. $A n \mathrm{GOx}-\mathrm{I} 489 \mathrm{~K}$ was readily modified by arPES, which enables the enzyme to exhibit MTT system-dependent dye-mediated glucose dehydrogenase activity and an electrochemical response on the electrode upon the addition of glucose. These properties were distinct from those of PES-modified AnGOx-WT. The enzyme electrode with PES-modified $A n G O x-I 489 \mathrm{~K}$ showed a response at a potential as low as $0 \mathrm{mV}$ vs. $\mathrm{Ag} / \mathrm{AgCl}$, which enabled the elimination of an effect from electrochemically-active interference. Additionally, since a direct electron transfer-type sensor does not require a free electron mediator, we are able to simplify the architecture of a sensor.

In conclusion, the engineered GOx we show in this case has the advantage of quasi-DET-ability in addition to its original functionality (high specificity and activity). This achievement realized a revived version of the "gold standard" glucose sensing enzyme, GOx, which has been used in the first-generation and second-generation principles based on glucose sensors, which will be available for the "2.5th generation" based glucose sensors. The addition of quasi-DET ability to GOx make 
this enzyme the invaluable material to construct a mediator-free type glucose sensor strip, and an ingredient impact-free CGM sensor.

Supplementary Materials: Supplementary Materials can be found at http://www.mdpi.com/1422-0067/21/3/1137/s1.

Author Contributions: Conceptualization, W.T. and K.S. Experimental design, Y.T-I.., J.O.-S., and K.M. Data acquisition, N.S., Y.T.-I., J.O., K.K., and K.M. Data analysis and interpretation, N.S., J.L., Y.T.-I., K.K., K.M., W.T., and K.S. Writing—original draft preparation, J.L. and N.L. Writing-review and editing, K.S. All authors have read and agreed to the published version of the manuscript.

Funding: This research received no external funding.

Acknowledgments: The authors appreciate Dojindo Laboratories Co. Ltd. (Kumamoto, Japan) very much for their kind supply of arPES.

Conflicts of Interest: The authors declare no conflict of interest.

\section{Abbreviations}

$\begin{array}{ll}\text { FAD } & \text { Flavin adenine dinucleotide } \\ \text { GOx } & \text { Glucose oxidase } \\ \text { GDH } & \text { Glucose dehydrogenase } \\ \text { DET } & \text { Direct electron transfer } \\ \text { MTT } & \text { 3-(4,5-Dimethyl-2-thiazolyl)-2,5-diphenyl-2H-tetrazolium bromide } \\ \text { PES } & \text { Phenazine ethosulfate } \\ \text { PMS } & \text { Phenazine methosulfate }\end{array}$

\section{References}

1. Clark, L.C.; Lyons, C. Electrode systems for continuous monitoring in cardiovascular surgery. Ann. N. Y. Acad. Sci. 1962, 102, 29-45. [CrossRef] [PubMed]

2. Cass, A.E.G.; Davis, G.; Francis, G.D.; Hill, H.A.O.; Aston, W.J.; Higgins, I.J.; Plotkin, E.V.; Scott, L.D.L.; Turner, A.P.F. Ferrocene-Mediated enzyme electrode for amperometric determination of glucose. Anal. Chem. 1984, 56, 667-671. [CrossRef]

3. Ferri, S.; Kojima, K.; Sode, K. Review of glucose oxidases and glucose dehydrogenases: A bird's eye view of glucose sensing enzymes. J. Diabetes Sci. Technol. 2011, 5, 1068-1076. [CrossRef] [PubMed]

4. Tremey, E.; Stines-Chaumeil, C.; Gounel, S.; Mano, N. Designing an $\mathrm{O}_{2}$-insensitive glucose oxidase for improved electrochemical applications. ChemElectroChem 2017, 4, 2520-2526. [CrossRef]

5. Horaguchi, Y.; Saito, S.; Kojima, K.; Tsugawa, W.; Ferri, S.; Sode, K. Engineering glucose oxidase to minimize the influence of oxygen on sensor response. Electrochim. Acta 2014, 126, 158-161. [CrossRef]

6. Gutierrez, E.A.; Mundhada, H.; Meier, T.; Duefel, H.; Bocola, M.; Schwaneberg, U. Reengineered glucose oxidase for amperometric glucose determination in diabetes analytics. Biosens. Bioelectron. 2013, 50, 84-90. [CrossRef]

7. Horaguchi, Y.; Saito, S.; Kojima, K.; Tsugawa, W.; Ferri, S.; Sode, K. Construction of mutant glucose oxidases with increased dye-mediated dehydrogenase activity. Int. J. Mol. Sci. 2012, 13, 14149-14157. [CrossRef]

8. Chaubey, A.; Malhotra, B.D. Mediated biosensors. Biosens. Bioelectron. 2002, 17, 441-456. [CrossRef]

9. Lee, I.; Loew, N.; Tsugawa, W.; Lin, C.; Probst, D.; La Belle, J.T.; Sode, K. The electrochemical behavior of a FAD dependent glucosedehydrogenase with direct electron transfer subunit by immobilizationon self-assembled monolayers. Bioelectrochemistry 2018, 121, 1-6. [CrossRef]

10. Bartlett, P.N.; Al-Lolage, F.A. There is no evidence to support literature claims of direct electron transfer (DET) for native glucose oxidase (GOx) at carbon nanotubes or graphene. J. Electroanal. Chem. 2018, 819, 26-37. [CrossRef]

11. Wilson, G.S. Native glucose oxidase does not undergo direct electron transfer. Biosens. Bioelectron. 2016, 82, Vii-Viii. [CrossRef] [PubMed]

12. Vogt, S.; Schneider, M.; Schafer-Eberwein, H.; Noll, G. Determination of the $\mathrm{pH}$ dependent redox potential of glucose oxidase by spectroelectrochemistry. Anal. Chem. 2014, 86, 7530-7535. [CrossRef]

13. Sampath, S.; Lev, O. Renewable, reagentless glucose sensor based on a redox modified enzyme and carbon-silica composite. Electroanalysis 1996, 8, 1112-1116. [CrossRef] 
14. Schuhmann, W. Electron-transfer pathways in amperometric biosensors. Ferrocene-modified enzymes entrapped in conducting-polymer layers. Biosens. Bioelectron. 1995, 10, 181-193. [CrossRef]

15. Badia, A.; Carlini, R.; Fernandez, A.; Battaglini, F.; Mikkelsen, S.R.; English, A.M. Intramolecular electron-transfer rates in ferrocene-derivatized glucose-oxidase. J. Am. Chem. Soc. 1993, 115, 7053-7060. [CrossRef]

16. Ryabov, A.D.; Trushkin, A.M.; Baksheeva, L.I.; Gorbatova, R.K.; Kubrakova, I.V.; Mozhaev, V.V.; Gnedenko, B.B.; Levashov, A.V. Chemical attachment of organometallics to proteins in reverse micelles. Angew. Chem. Int. Ed. 1992, 31, 789-791. [CrossRef]

17. Schuhmann, W.; Ohara, T.J.; Schmidt, H.L.; Heller, A. Electron-transfer between glucose-oxidase and electrodes via redox mediators bound with flexible chains to the enzyme surface. J. Am. Chem. Soc. 1991, 113, 1394-1397. [CrossRef]

18. Bartlett, P.N.; Bradford, V.Q.; Whitaker, R.G. Enzyme electrode studies of glucose-oxidase modified with a redox mediator. Talanta 1991, 38, 57-63. [CrossRef]

19. Degani, Y.; Heller, A. Direct electrical communication between dhemically modified enzymes and metal-electrodes. 2. Methods for bonding electron-transfer relays to glucose-oxidase and D-amino-acid oxidase. J. Am. Chem. Soc. 1988, 110, 2615-2620. [CrossRef]

20. Degani, Y.; Heller, A. Direct electrical communication between chemically modified enzymes and metal-electrodes. 1. Electron-transfer from glucose-oxidase to metal-electrodes via electron relays, bound covalently to the enzyme. J. Phys. Chem. 1987, 91, 1285-1289. [CrossRef]

21. Bartlett, P.N.; Whitaker, R.G.; Green, M.J.; Frew, J. Covalent binding of electron relays to glucose-oxidase. J. Chem. Soc. Chem. Comm. 1987, 1603-1604. [CrossRef]

22. Ban, K.; Ueki, T.; Tamada, Y.; Saito, T.; Imabayashi, S.; Watanabe, M. Fast electron transfer between glucose oxidase and electrodes via phenothiazine mediators with poly(ethylene oxide) spacers attached to the enzyme surface. Electrochem. Commun. 2001, 3, 649-653. [CrossRef]

23. Ryabova, E.S.; Goral, V.N.; Csoregi, E.; Mattiasson, B.; Ryabov, A.D. Coordinative approach to mediated electron transfer: Ruthenium complexed to native glucose oxidase. Angew. Chem. Int. Ed. 1999, 38, 804-807. [CrossRef]

24. Bartlett, P.N.; Booth, S.; Caruana, D.J.; Kilburn, J.D.; Santamaria, C. Modification of glucose oxidase by the covalent attachment of a tetrathiafulvalene derivative. Anal. Chem. 1997, 69, 734-742. [CrossRef]

25. Krikstopaitis, K.; Kulys, J.; Tetianec, L. Bioelectrocatalytical glucose oxidation with phenoxazine modified glucose oxidase. Electrochem. Commun. 2004, 6, 331-336. [CrossRef]

26. Hatada, M.; Loew, N.; Inose-Takahashi, Y.; Okuda-Shimazaki, J.; Tsugawa, W.; Mulchandani, A.; Sode, K. Development of a glucose sensor employing quick and easy modification method with mediator for altering electron acceptor preference. Bioelectrochemistry 2018, 121, 185-190. [CrossRef]

27. Hiraka, K.; Kojima, K.; Tsugawa, W.; Asano, R.; Ikebukuro, K.; Sode, K. Rational engineering of Aerococcus viridans L-lactate oxidase for the mediator modification to achieve quasi-direct electron transfer type lactate sensor. Biosens. Bioelectron. 2019, 151, 111974. [CrossRef]

28. Yoshida, H.; Sakai, G.; Mori, K.; Kojima, K.; Kamitori, S.; Sode, K. Structural analysis of fungus-derived FAD glucose dehydrogenase. Sci. Rep. 2015, 5, 13498. [CrossRef]

29. Thompson, J.D.; Higgins, D.G.; Gibson, T.J. CLUSTAL W: Improving the sensitivity of progressive multiple sequence alignment through sequence weighting, position-specific gap penalties and weight matrix choice. Nucleic Acids Res. 1994, 22, 4673-4680. [CrossRef]

30. Okurita, M.; Suzuki, N.; Loew, N.; Yoshida, H.; Tsugawa, W.; Mori, K.; Kojima, K.; Klonoff, D.C.; Sode, K. Engineered fungus derived FAD-dependent glucose dehydrogenase with acquired ability to utilize hexaammineruthenium(III) as an electron acceptor. Bioelectrochemistry 2018, 123, 62-69. [CrossRef]

(C) 2020 by the authors. Licensee MDPI, Basel, Switzerland. This article is an open access article distributed under the terms and conditions of the Creative Commons Attribution (CC BY) license (http://creativecommons.org/licenses/by/4.0/). 